\title{
DC Conductivity and Peierls Instability in the Quasi-One-Dimensional Organic Charge Density Wave Conductor (Fluoranthene) ${ }_{2} \mathrm{X}$
}

\author{
W. Brütting, W. Riess, M. Schwoerer \\ Physikalisches Institut und Bayreuther Institut für Makromolekülforschung (BIMF), \\ Universität Bayreuth, Postfach 1012 51, \\ D-8580 Bayreuth, Germany
}

\begin{abstract}
Due to the high anisotropy of the dc conductivity $\left(\sigma_{\|} / \sigma_{\perp} \approx 10^{4}\right)$ the organic conductor (fluoranthene) ${ }_{2} \mathrm{X}$ can be regarded as a model system for studying the Peierls instability in quasi-one-dimensional systems. The temperature dependence of the dc conductivity $\sigma_{\|}(T)$ along the highly conducting crystal axis exhibits the typical behaviour of a quasi-one-dimensional metal with a Peierls transition at about $180 \mathrm{~K}$ to a charge density wave (CDW) ground state. As expected for a highly one-dimensional conductor the exact transition temperature depends on three-dimensional coupling effects and therefore on the size of the counterion $\mathrm{X}^{-}=\mathrm{PF}_{6}^{-}, \mathrm{AsF}_{6}^{-}, \mathrm{SbF}_{6}^{-}$. Above the Peierls transition $\sigma_{\|}(T)$ can be described quantitatively within a model of CDW fluctuations leading to a pseudo gap in the electronic density of states. Below, the existence of a real energy gap at the Fermi level with a BCS-like temperature dependence determines the charge transport over more than eight orders of magnitude in the electrical resistance. For the intrinsic energy gaps $2 \Delta(0)$, which characterize the ground state of the Peierls semiconductor, values of $120-180 \mathrm{meV}$ have been found for different crystals.
\end{abstract}

Keywords: Low-dimensional systems; Peierls instability; Charge Density Waves

\section{Introduction}

Theoretical investigations of one-dimensional conductors — started well before quasione-dimensional crystals were prepared experimentally - have demonstrated that their electronic properties differ considerably from those materials, in which two- or threedimensional motion of electrons is possible.

Already in the year 1955 Peierls theoretically proposed a metal-insulator transition for a coupled one-dimensional electron-phonon system [1]. When the temperature is lowered a lattice distortion with a wave number equal to twice the Fermi momentum $k_{F}$ of the electronic system splits the partially filled conduction band into completely filled and empty subbands separated by an energy gap $2 \Delta$, thus turning the electronic properties of the crystal from metallic to insulating. This periodic distortion of the 
lattice is accompanied by a modulation of the electronic charge density, a so-called charge density wave (CDW).

These ideas resurfaced when the first materials with highly anisotropic crystal and electronic structures became available in the early 1970s. Meanwhile several inorganic and organic compounds have been found exhibiting electrical properties characteristic of one dimension [2]. Besides CDWs other collective phenomena like superconductivity and spin density waves (SDW) have been observed in these new substances [3].

The fluoranthene radical cation salts belong to the class of one-dimensional conductors with a Peierls transition to a CDW ground state. Originally the observation of this collective transport phenomenon was limited to inorganic systems such as the transition metal compounds $\mathrm{NbSe}_{3}, \mathrm{TaS}_{3},\left(\mathrm{TaSe}_{4}\right)_{2} \mathrm{I}$ and $\left(\mathrm{NbSe}_{4}\right)_{3.33} \mathrm{I}$ and the blue molybdenum bronzes like $\mathrm{K}_{0.30} \mathrm{MoO}_{3}$, but meanwhile it has been found in materials such as TTFTCNQ, where the charge transport is determined by organic molecules, too [4].

In this paper we present an in depth analysis of the Peierls instability in the quasione-dimensional organic CDW conductor (fluoranthene) ${ }_{2} \mathrm{X}\left(\mathrm{X}^{-}=\mathrm{PF}_{6}^{-}, \mathrm{AsF}_{6}^{-}, \mathrm{SbF}_{6}^{-}\right)$. For this purpose we have investigated the dc conductivity of the system and compared our data with the predictions of theoretical models developed for one-dimensional conductors undergoing a Peierls transition [5].

The existence of a CDW ground state in (fluoranthene $)_{2} \mathrm{X}$ below the Peierls transition was confirmed by the observation of nonlinear and frequency dependent conductivity, conductivity noise and metastability phenomena [6]. Thus the fluoranthene radical cation salts seem to be an exception of the general feature, that systems with dimerized donor or acceptor stacks such as the salts of the (TMTSF) $)_{2} \mathrm{X}$-family exhibit a spin density wave instability at low temperatures [3].

\section{Crystal structure}

Single crystals of (fluoranthene) $)_{2} \mathrm{X}$ were obtained by anodic oxidation of the aromatic hydrocarbon fluoranthene $\left(\mathrm{C}_{16} \mathrm{H}_{10}\right)$ - abbreviated in the following as $\mathrm{FA}$ - in the presence of suitable anions $\left(\mathrm{X}^{-}=\mathrm{PF}_{6}^{-}, \mathrm{AsF}_{6}^{-}, \mathrm{SbF}_{6}^{-}\right)$as described elsewhere [7].

Figure 1 shows the crystal structure of $(\mathrm{FA})_{2} \mathrm{X}$ at room temperature for the counterion $\mathrm{X}^{-}=\mathrm{PF}_{6}^{-}$. Typical of the system is the linear arrangement of FA-molecules in stacks of dimer radical cations $(\mathrm{FA})_{2}^{\odot+}$ along the crystallographic a-axis segregated by counterions $\mathrm{X}^{-}$. Responsible for the electronic and magnetic properties are these FA-dimers, which carry one elementary electric charge and spin $\frac{1}{2}$ due to the unpaired electron, whereas the centrosymmetric anions $\mathrm{X}^{-}$have closed electron shells and therefore spin 0 .

The mean intermolecular distance of the FA-molecules along the crystallographic a-axis $(\mathrm{a}=3.3 \AA$ ), which is less than twice the Van der Waals radius of the molecule, ensures a strong overlap of the electronic $\pi$-orbitals of neighbouring molecules within the FA-stack. Due to the considerably larger lattice constants $b=12.57 \AA$ and $c=14.77 \AA$, as well as the spatial direction of the $\pi$-orbitals parallel to the a-axis, the electronic overlap perpendicular to the FA-stack is far less, thus giving rise to a high anisotropy of the material.

Together with the charge transfer of one electron per FA-dimer these overlapping electron wave functions lead to the formation of a half-filled one-dimensional conduction band, which can be well described within a tight-binding model [8]. In this model the 
energy dispersion relation is given by:

$$
E_{k}=-2 t_{\|} \cos (k a)
$$

with a longitudinal bandwidth $w_{\|}=4 t_{\|}=1.5 \mathrm{eV}$ derived from polarized reflectance spectra [9].

The transversal bandwidth $w_{\perp}$ can be estimated from the ratio of the conductivities parallel and perpendicular to the highly conducting a-axis by means of:

$$
w_{\perp}=w_{\|}\left(\frac{a_{\|}}{a_{\perp}}\right)\left(\frac{\sigma_{\perp}}{\sigma_{\|}}\right)^{1 / 2}
$$

For $(\mathrm{FA})_{2} \mathrm{PF}_{6}$ we have found $\sigma_{\|} / \sigma_{\perp} \approx 10^{4}$ leading to $w_{\perp} \approx 10 \mathrm{meV}$. Therefore the system is characterized by highly anisotropic crystal and electronic structures, which lead to the observed anisotropy of electrical transport properties.

\section{Experimental}

The dc conductivity $\sigma_{\|}(T)$ of freshly grown $(\mathrm{FA})_{2} \mathrm{X}$ single crystals parallel to the highly conducting a-axis was investigated from room temperature down to $4 \mathrm{~K}$ using two different experimental methods. From $300 \mathrm{~K}$ to about $30 \mathrm{~K}$ the electrical resistance was measured by a four probe technique with a current source (Keithley Model 220) and a nanovoltmeter (Keithley Model 181) or an electrometer (Keithley Model 617), which allow the measurement of resistances from $10^{-6}-10^{10} \Omega$. At temperatures below, where the sample resistance exceeded $10^{10} \Omega$, a two point configuration with the electrometer as source and sense device was used, making measurements of resistances up to $10^{16} \Omega$ possible. With this technique we have ensured that contact resistances were negligible as compared to the sample resistance, which is in general true for $(\mathrm{FA})_{2} \mathrm{X}$ at temperatures below $50 \mathrm{~K}$.

The measurements were performed on single crystals of $(\mathrm{FA})_{2} \mathrm{X}$ from different batches with typical dimensions of $3 \times 0.1 \times 0.1 \mathrm{~mm}^{3}$. Electrical contacts were made by mechanically clamping fine wires to gold pads evaporated on the crystal [10]. Homogeneous current injection, which is absolutely necessary due to the high anisotropy of the system, was achieved by covering the crystal ends also with gold. With this mounting technique we did not find any sudden jumps in the resistance upon cooling down the sample.

In former investigations, where we used a different mounting technique fixing fine wires to the crystal with conducting paste (gold or graphite paint), we always registered nonreproducible resistance jumps when cooling the crystal. Similar observations were made on other organic conductors, too $[10,11]$. There the sudden resistance jumps were ascribed to microcracks in the crystal, produced by mechanical strains originating from contacts with conducting paste.

The jumps observed on $(\mathrm{FA})_{2} \mathrm{X}$ crystals, however, were accompanied by a considerable decrease in the optical reflectivity, indicating that mechanical strains can lead to a microscopic change of the electronic orbital overlap, which causes a reduction of the macroscopic conductivity. 


\section{Results and discussion}

In the following the temperature dependence of the de conductivity $\sigma_{\|}(T)$ along the crystallographic a-axis will be presented for $(\mathrm{FA})_{2} \mathrm{PF}_{6}$, where we have achieved the highest crystal quality. Thereafter we will describe the observed dependency within theoretical models and finally discuss the influence of different counterions on the phase transition.

\subsection{DC conductivity of $(\mathrm{FA})_{2} \mathrm{PF}_{6}$ : survey}

Between room temperature and $4 \mathrm{~K}$ the dc conductivity of $(\mathrm{FA})_{2} \mathrm{PF}_{6}$ varies over more than 16 orders of magnitude with maximum values of $10^{2}-10^{3}(\Omega \mathrm{cm})^{-1}$ - depending on the crystal - at room temperature. At temperatures above $300 \mathrm{~K}$, a slow aging process is observed due to a chemical decomposition of the substance.

Figure 2 shows an Arrhenius plot of $\sigma_{\|}(T)$ between 300 and $20 \mathrm{~K}$. This plot can be clearly divided into four separate temperature regions:

- A. High temperature range $(300-182 \mathrm{~K})$ :

Due to the relatively high conductivities one also speaks of the "metallic range", though, however, no real metallic temperature dependence of the conductivity is observed (this would mean: $d \sigma_{\|} / d T<0$ ). In this range the system can be characterized as a quasi-one-dimensional metal, in which fluctuations lead to a pseudo gap in the electronic density of states.

- Peierls transition $\left(T_{P}=182 \mathrm{~K}\right)$ :

At $182 \mathrm{~K}$ we observe a phase transition of second order, which is also seen in DSC (differential scanning calorimetry) [12] and static magnetic susceptibility measurements [13]. This is the Peierls transition leading to the CDW ground state of the system. The transition temperature can be precisely determined from the logarithmic derivative $d\left(\log \sigma_{\|}\right) / d(1 / T)$ in figure 3 , which shows a discontinuity at the Peierls transition caused by an abrupt change of the curvature of $\sigma_{\|}(T)$.

- B. Intermediate range $(182-120 \mathrm{~K})$ :

Below the Peierls transition a strong decrease of conductivity is observed due to the gradual opening of a real energy gap at the Fermi level with a modified BCS-like temperature dependence.

- C. Semiconducting range $(120-50 \mathrm{~K})$ :

Here the energy gap is almost completely open and one therefore measures thermally activated conductivity with activation energies $\Delta(0)=60-90 \mathrm{meV}$ for crystals from different batches.

- D. Low temperature range $(\mathrm{T}<50 \mathrm{~K})$ :

Below approximately $50 \mathrm{~K}$ deviations from the thermally activated behaviour indicate that impurity levels within the gap contribute to the conductivity.

In the following we will analyze the observed temperature dependence of the dc conductivity in the ranges $\mathrm{A}, \mathrm{B}$ and $\mathrm{C}(300-50 \mathrm{~K})$, where the influence of defects is not dominant, with theoretical models developed for quasi-one-dimensional systems with a Peierls transition. The CDW ground state in the intermediate and semiconducting range can thereby be treated as a unity. 


\subsection{Metallic range of $(\mathrm{FA})_{2} \mathrm{PF}_{6}$}

Already from earlier experiments there has been strong evidence for a metallic character of $(\mathrm{FA})_{2} \mathrm{PF}_{6}$ in the high temperature range. In polarized optical reflectance spectra a reflectivity of almost $100 \%$ for light polarized parallel to the FA stacking axis together with a pronounced plasma edge at $\hbar \omega_{p l} \approx 1.5 \mathrm{eV}$ has been found [9]. Furthermore magnetic resonance experiments revealed a Korringa law for the longitudinal nuclear spin relaxation time $T_{1}$ in the temperature range above the Peierls transition [14]:

$$
\frac{1}{T_{1}} \propto T
$$

In contrast to these results, which are typical for metals, is the observed non-metallic temperature dependence of the dc conductivity, which, however, can be explained as a consequence of the high one-dimensionality of the system.

Theoretical calculations of Lee, Rice and Anderson [15] have shown that in a onedimensional electron-phonon system thermal fluctuations lead to a pseudo energy gap in the electronic density of states at the Fermi level. This means that with decreasing temperature a dip in the electronic density of states is observed in the vicinity of the Fermi energy $\left(\left|E-E_{F}\right|<\Delta(0)\right)$. Although $\mathcal{D}(E, T)$ remains finite for $T>0$, the significant reduction below the high temperature metallic density of states has an influence on macroscopic transport properties.

Recently Johnston et al. [16] were able to show that for a calculation of macroscopic transport properties the pseudo gap in the electronic density of states $\mathcal{D}(E, T)$ can be replaced by an effective uniform, but temperature dependent energy gap $2 \Delta_{\text {eff }}(T)$, which is displayed in figure 4 . They introduced a model to describe the temperature dependence of the electrical resistance of the quasi-one-dimensional CDW conductor $\left(\mathrm{TaSe}_{4}\right)_{2} \mathrm{I}$ above the Peierls transition by the following equation:

$$
R(T)=R_{0}+A \cdot T^{B} \cdot\left[\exp \left\{\Delta_{e f f}(T) / k_{B} T\right\}+1\right]
$$

This equation can be derived from the Boltzmann equation for the electrical conductivity of a one-dimensional semiconductor with a band gap $2 \Delta$ :

$$
\sigma(T)=\frac{4 e^{2} n_{c}}{\pi \hbar} \int_{\Delta}^{\infty} \tau(\epsilon) v(\epsilon)\left(-\frac{\partial f_{F D}}{\partial \epsilon}\right) d \epsilon
$$

where $\tau$ and $v$ denote the scattering time and drift velocity of the charge carriers and $f_{F D}(\epsilon)=\left[\exp \left(\epsilon / k_{B} T\right)+1\right]^{-1}$ is the Fermi-Dirac distribution for a Fermi energy fixed at $E=0 . n_{c}$ represents the number of conducting chains per unit area. By taking an energy independent mean free path of electrons $l=\tau v$ and replacing $2 \Delta$ by the effective uniform gap $2 \Delta_{e f f}(T)$ one yields:

$$
\sigma(T)=\frac{4 e^{2} n_{c} l}{\pi \hbar}\left[\exp \left\{\Delta_{e f f}(T) / k_{B} T\right\}+1\right]^{-1}
$$

Equation (4) follows, when a power law for $l(T) \propto T^{-B}$ is assumed. $R_{0}$ merely represents a phenomenological offset, which has been introduced by Johnston to account for the sample geometry. 
In figure 5 the Johnston equation (4) was fitted to the high temperature $R(T)$ data from 182 to $290 \mathrm{~K}$. Thereby we have achieved good agreement between theory and experiment for $T>200 \mathrm{~K}$.

Of physical importance is the mobility exponent $\mathrm{B}$, for which fits for different crystals have yielded values from $0.8-1.1$, being typical for metals at high temperatures. This indicates that $(\mathrm{FA})_{2} \mathrm{PF}_{6}$ in its high temperature phase possesses metallic character. $\mathrm{A}$ metal-like temperature dependence of the dc conductivity, however, is suppressed by fluctuations.

With the knowledge of the effective uniform gap $2 \Delta_{\text {eff }}(T)$ we can estimate the mean free path of electrons at $T=290 \mathrm{~K}$ from equation $(6)$ with $\sigma_{\|}(290 \mathrm{~K})=1000(\Omega \mathrm{cm})^{-1}$ and $n_{c}=\frac{2}{b c}=1.1 \times 10^{14} \mathrm{~cm}^{-2}$ yielding $l=12.3 \AA$, which is in good agreement with values calculated from optical reflectance spectra [9] and magnetic resonance experiments [17].

For the deviations below $T=200 \mathrm{~K}$ two facts may be responsible. Just like in other CDW conductors it is likely that in the vicinity of the phase transition two- or three-dimensional coupling effects of neighbouring chains become important, leading to deviations from the above equation. Besides, in the special case of $(\mathrm{FA})_{2} \mathrm{PF}_{6}$ a structural phase transition from space group $A 2 / m$ to $P 2_{1} / c$ at $206 \mathrm{~K}$ detected by DSC [12] and xray analysis [7] contributes an additional scattering mechanism of charge carriers below this temperature.

\subsection{Ground state of $(\mathrm{FA})_{2} \mathrm{PF}_{6}$}

According to the early theory of Peierls the ground state of a one-dimensional metal is an insulating (respectively semiconducting) one, due to the existence of an energy gap at the Fermi level.

Rice and Strssler [18] have treated the Peierls transition in a strictly one-dimensional electron-phonon system within the mean-field theory. They have shown that the temperature dependence of the energy gap $2 \Delta(T)$ can be derived from the following expression:

$$
\frac{1}{\lambda}=\int_{0}^{E_{F}} \tanh \left(\frac{\sqrt{\epsilon^{2}+\Delta^{2}(T)}}{2 k_{B} T}\right) \frac{d \epsilon}{\sqrt{\epsilon^{2}+\Delta^{2}(T)}}
$$

In this equation $\lambda$ denotes the so-called dimensionless electron-phonon coupling constant, given by:

$$
\lambda=\frac{\left|g\left(k=k_{F}, q=2 k_{F}\right)\right|^{2} \mathcal{D}\left(E_{F}\right)}{\hbar \omega_{2 k_{F}}},
$$

where $g$ is the matrix element for the scattering process of electrons with wave number $k=k_{F}$ and phonons of $q=2 k_{F}, \omega_{2 k_{F}}$ the unperturbed phonon frequency of the metallic range for phonons with $q=2 k_{F}$ and $\mathcal{D}\left(E_{F}\right)$ the density of states at the Fermi level.

The formal structure of equation (7) is identical to the one for the energy gap of a superconductor in the BCS-theory of superconductivity, if $\lambda$ is replaced by $U \cdot \mathcal{D}\left(E_{F}\right)$, where $U$ represents the effective electron-electron interaction potential of a superconductor, and the upper integration limit by the Debye energy $\hbar \omega_{D}$. While the ground state of a superconductor is characterized by the existence of electron-electron pairs (Cooper pairs) with momentum $k=0$, the ground state of the Peierls semiconductor can be regarded as a condensate of electron-hole pairs with $k=2 k_{F}$. 
The mean-field theory for a half-filled tight-binding band furthermore yields expressions for the Peierls transition temperature $T_{P}^{M F}[18]$ :

$$
k_{B} T_{P}^{M F}=2.26 E_{F} \exp (-1 / \lambda)
$$

and the zero temperature energy gap $2 \Delta(0)$ :

$$
2 \Delta(0)=8 E_{F} \exp (-1 / \lambda)
$$

From these equations follows the fundamental BCS relation between the energy gap $2 \Delta(0)$ and the transition temperature $T_{P}^{M F}$ :

$$
2 \Delta(0)=3.52 k_{B} T_{P}^{M F}
$$

In the mean-field theory, however, one neglects the effects of thermodynamic fluctuations, which for a strictly one-dimensional system would preclude the occurrence of a phase transition at finite temperature. In real quasi-one-dimensional systems a nonvanishing three-dimensional coupling of conducting chains partially suppresses the effect of such fluctuations, so that a Peierls transition can take place at a finite temperature $T_{P}$ with:

$$
0<T_{P}<T_{P}^{M F}
$$

Although a theoretical treatment of the Peierls transition, taking into account both fluctuations and three-dimensional coupling effects, requires rather sophisticated methods [19], the mean-field results can give at least qualitative insight into the ground state of a quasi-one-dimensional conductor.

At low temperatures thermal fluctuations disappear, and quantum effects are negligible due to the large effective mass $M^{*}$ of the CDW condensate, which is of the order of $10^{3} \cdot m^{*}\left(m^{*} \approx m_{e}\right.$ is the effective band mass of the conduction electrons) [20]. Consequently, the low temperature properties of the system are well described by the mean-field results. In particular one expects the real Peierls gap at $T=0$ to be approximately the same as the mean-field gap:

$$
\Delta(0) \approx \Delta^{M F}(0)
$$

At higher temperatures, especially in the vicinity of $T_{P}^{M F}$, fluctuations are not negligible and the real Peierls transition occurs at a significantly lower temperature $T_{P}$, as shown in figure 6 .

Therefore the energy gap $2 \Delta(0)$ at $T=0$ and the Peierls transition temperature $T_{P}$ do not satisfy the fundamental BCS relation (equation (11)). Instead of the proportionality constant 3.52 we find for $(\mathrm{FA})_{2} \mathrm{PF}_{6}$ from the measured activation energies $\Delta(0)=60-90 \mathrm{meV}$ (yielding mean-field transition temperatures of $T_{P}^{M F}=400-600 \mathrm{~K}$ ) and the observed Peierls transition at $T_{P}=182 \mathrm{~K}$ :

$$
2 \Delta(0)=(7.7-11.5) k_{B} T_{P}
$$

For the dimensionless electron-phonon coupling constant we have calculated from equation (10) with a Fermi energy given by the tight-binding model as $E_{F}=t_{\|}=0.37 \mathrm{eV}$ :

$$
\lambda=0.31-0.36
$$


which are typical values for CDW conductors [21, 22].

By postulating that the temperature dependence of the energy gap $2 \Delta(T)$ in $(\mathrm{FA})_{2} \mathrm{PF}_{6}$ below $182 \mathrm{~K}$ is of the modified BCS-type (as displayed in figure 6 ) we were able to describe the observed $R(T)$ dependency quantitatively with the analogon of equation (4), in which the offset $R_{0}$ has been neglected and the Fermi-Dirac distribution replaced by Maxwell-Boltzmann statistics:

$$
R(T)=A \cdot T^{B} \cdot \exp \left\{\Delta(T) / k_{B} T\right\}
$$

For a calculation of $\Delta(T)$ we have taken for $\Delta(0)$ the experimentally obtained values in the semiconducting range and scaled down the original BCS-gap to a smaller critical temperature $T_{P}^{*}$, for which the energy gap vanishes (confer figure 6 ). As we shall see later, this temperature $T_{P}^{*}$ and the observed transition temperature $T_{P}$ are not completely identical, but differ by about $8 \mathrm{~K}$.

Figure 7 shows the excellent fit of equation (16) to the $R(T)$ data below the Peierls transition over more than eight orders of magnitude in the electrical resistance. Remarkable thereby is the good agreement of the exponent $B=0.7-1.0$ for different crystals with the values obtained for the high temperature range.

\subsection{Energy gap in $(\mathrm{FA})_{2} \mathrm{PF}_{6}$}

We have shown in the last two paragraphs that the dc conductivity of $(\mathrm{FA})_{2} \mathrm{PF}_{6}$ can be described quantitatively in a wide temperature range from room temperature down to $50 \mathrm{~K}$, if one knows the functional form of only two physical quantities. These are the effective uniform gap for the metallic range (caused by fluctuations) and the real energy gap in the ground state, which is the order parameter of the Peierls transition. The temperature dependence of $\Delta_{e f f}(T)$ and $\Delta(T)$ is determined by the activation energy $\Delta(0)$ and the phase transition temperature $T_{P}$, which can both be easily measured. These two energy gaps, which have already been used for the fits from above, are displayed in figure 8 .

According to this figure the observed Peierls transition takes place at a temperature $T_{P}=182 \mathrm{~K}$, where the effective gap with only a weak temperature dependence passes into the strongly temperature dependent Peierls gap, which vanishes at a slightly higher temperature $T_{P}^{*}=190 \mathrm{~K}$. This figure also explains the observed singularity in the derivative of $\sigma_{\|}(T)$ at $T_{P}$ (confer figure 3) as a consequence of the different curvatures of $\Delta_{\text {eff }}(T)$ and $\Delta(T)$. Our results on the energy gap confirm calculations for the effective gap in the metallic range from static magnetic susceptibility data [13]. Moreover, dc conductivity measurements yield precise information below the phase transition, where susceptibility data soon become rather inaccurate.

\subsection{Peierls transition in $(\mathrm{FA})_{2} \mathrm{X}$}

As already mentioned, a Peierls transition at a finite temperature $T_{P}>0$ can only occur in the presence of a non-vanishing interaction between the conducting chains of a quasi-one-dimensional conductor. Consequently the exact phase transition temperature depends on the magnitude of these three-dimensional coupling effects.

In order to investigate this influence in our system $(\mathrm{FA})_{2} \mathrm{X}$ we have measured the dc conductivity of crystals with the anions $\mathrm{X}^{-}=\mathrm{PF}_{6}^{-}, \mathrm{AsF}_{6}^{-}, \mathrm{SbF}_{6}^{-}$. For counterions other 
than $\mathrm{PF}_{6}^{-}$we observe a similar behaviour of $\sigma_{\|}(T)$, however, as expected for a highly onedimensional conductor the Peierls transition temperature depends on three-dimensional coupling effects through the size of the counterion. With increasing size of the counterion we observe a shift of the phase transition towards lower temperatures indicating a weaker three-dimensional coupling. The respective lattice parameters together with the measured phase transition temperatures are listed in table 1.

In theoretical treatments of the Peierls transition in quasi-one-dimensional conductors three principal mechanisms of interchain coupling are discussed [19, 21]. First the Coulomb interaction between CDWs on neighbouring chains, second tunneling of electrons between adjacent chains, described by a finite transfer integral $t_{\perp}$, and finally the dependence of the phonon frequencies on the transversal components of the wave vector, originating from interactions between ions on adjacent chains.

These theories show that the real Peierls transition temperature $T_{P}$ is a monotonously increasing function of the interchain coupling. Furthermore, even in highly onedimensional systems like $(\mathrm{FA})_{2} \mathrm{X}$ a small interchain coupling is sufficient for a Peierls transition at relatively high temperatures of the order of $T_{P} \approx(0.3-0.5) T_{P}^{M F}$.

Estimates of the coupling constants indicate that three-dimensional coupling in $(\mathrm{FA})_{2} \mathrm{X}$ is weak in accordance with the observed high one-dimensionality of the system. Further investigations with different experimental methods are necessary, in order to identify the dominant coupling mechanism and to answer the question, why the Peierls transition in $(\mathrm{FA})_{2} \mathrm{X}$ crystals with the same counterion $\mathrm{X}^{-}$but different energy gaps $2 \Delta(0)$ always occurs at a fixed temperature $T_{P}$ (depending on the counterion) and does not scale with the mean-field transition temperature $T_{P}^{M F}$ (given by $\Delta(0)$ ).

\section{Conclusion}

Due to the crystal structure and the spatially directed electronic $\pi$-orbitals of the organic molecule fluoranthene we observe highly anisotropic electrical transport properties. The measured anisotropy of the dc conductivity of $\sigma_{\|} / \sigma_{\perp} \approx 10^{4}$ represents a relatively high value as compared to the other CDW conductors mentioned in the introduction, where ratios of $\sigma_{\|} / \sigma_{\perp} \approx 10-100$ are reported [20]. Therefore $(\mathrm{FA})_{2} \mathrm{X}$ can be regarded as a model system for studying the Peierls instability in quasi-one-dimensional conductors.

Our investigations have shown that the temperature dependence of the dc conductivity in the quasi-one-dimensional organic $\mathrm{CDW}$ conductor $(\mathrm{FA})_{2} \mathrm{X}\left(\mathrm{X}^{-}=\mathrm{PF}_{6}^{-}, \mathrm{AsF}_{6}^{-}\right.$, $\left.\mathrm{SbF}_{6}^{-}\right)$can be described quantitatively over a wide temperature range from 300 to about $50 \mathrm{~K}$, in which the electrical resistance varies over more than 8 orders of magnitude, within theoretical models developed for quasi-one-dimensional systems.

In the metallic range above the Peierls transition the conductivity can be described quantitatively by CDW fluctuations leading to a pseudo gap in the electronic density of states at the Fermi level. Our analysis of the conductivity based on this model has revealed a mean free path of electrons $l(T) \propto T^{-B}(B=0.8-1.1)$, which indicates that in a microscopic picture (FA) $)_{2} \mathrm{X}$ actually has metallic properties in its high temperature phase. Thus the observed non-metallic behaviour of the macroscopic conductivity $\sigma_{\|}(T)$ $\left(d \sigma_{\|}(T) / d T>0\right)$ clearly shows the strong impact of one-dimensional fluctuations in this temperature range.

Below the Peierls transition, in the CDW ground state the existence of an energy gap $2 \Delta(T)$ in the single particle excitation spectrum with a modified BCS-type temperature 
dependence determines the charge transport. We have found that for temperatures down to about $50 \mathrm{~K}$ the basic results of mean-field theory - originally developed for a strictly one-dimensional system without fluctuations - remain valid even for a real quasi-onedimensional conductor like $(\mathrm{FA})_{2} \mathrm{X}$. The observed transition temperature $T_{P}=182 \mathrm{~K}$, however, is significantly suppressed by fluctuations below the mean-field value $T_{P}^{M F}=$ $400-600 \mathrm{~K}$.

As expected for a highly one-dimensional conductor the transition temperature depends on three-dimensional coupling effects and therefore on the size of the counterion $\mathrm{X}^{-}$. In spite of the high one-dimensionality of $(\mathrm{FA})_{2} \mathrm{X}$, the observed Peierls transition occurs at a considerable fraction of the mean-field temperature $T_{P}^{M F}$, which is of the order of $T_{P} \approx(0.3-0.5) T_{P}^{M F}$.

As already mentioned we have found clear evidence for the existence of a CDW ground state in $(\mathrm{FA})_{2} \mathrm{X}$ below the Peierls transition. This broken symmetry ground state is characterized by the existence of collective transport phenomena unknown from usual metals or semiconductors. Among them are field and frequency dependent conductivity, conductivity noise and metastability phenomena. It is to expect that a coherent response of the CDW condensate in $(\mathrm{FA})_{2} \mathrm{X}$ crystals will be prevented by the high onedimensionality of this system. These results, however, will be presented in companion paper [23].

\section{Acknowledgements}

We thank J. Gmeiner for growing the single crystals, S. Karg for experimental assistance and D. Rainer (Theoretische Physik III, Universität Bayreuth) for stimulating discussions. This work was supported by BASF/BMFT and Fonds der Chemischen Industrie.

\section{References}

[1] R.E. Peierls, Quantum Theory of Solids (Clarendon, Oxford 1955), p. 108

[2] S. Kagoshima, H. Nagasawa and T. Sambongi, One-Dimensional Conductors (Springer, Berlin 1988)

[3] D. Jerome and H.J. Schulz, Adv. Phys. 31 (1982) 299; K. Bechgaard and D. Jerome, Physica Scripta T39 (1991) 37

[4] For a review of CDW conductors see for example: P. Monceau (Ed.), Electronic Properties of Inorganic Quasi-One-Dimensional Compounds I+II (Reidel, Dordrecht 1985); L.P. Gorkov and G. Grüner (Eds.), Charge Density Waves in Solids (North Holland, Amsterdam 1989)

[5] For reviews on the Peierls transition see for example:

L.N. Bulaevskii, Sov. Phys. Usp. 18 (1976) 131; G.A. Toombs, Phys. Rep. 40 (1978) 181

[6] W. Riess, W. Schmid, J. Gmeiner and M. Schwoerer, Synth. Met. 42 (1991) 2261; W. Riess, W. Brütting and M. Schwoerer, Mol. Cryst. Liq. Cryst., to be published (1992)

[7] V. Enkelmann, B.S. Morra, Ch. Kröhnke, G. Wegner and J. Heinze, Chem. Phys., 66 (1982) 303

[8] M. Mehring in: D. Jerome and L.G. Caron (Eds.), Low-Dimensional Conductors and Superconductors (Plenum, New York 1987), p. 185

[9] Th. Schimmel, B. Koch, H.P. Geserich and M. Schwoerer, Synth. Met., 33 (1989) 311 
[10] V.B. Ginodmann, A.V. Gudenko, P.A. Kononovich, V.N. Laukhin and I.F. Shchegolev, Sov. Phys. JETP 67 (1988) 1055

[11] W. Kang, S. Tomic, J.R. Cooper and D. Jerome, Phys. Rev. B 41 (1990) 4862; G. Mihaly, Y. Kim and G. Grüner, Phys. Rev. Lett. 66 (1991) 2806

[12] Th. Schimmel, W. Riess, G. Denninger and M. Schwoerer, Ber. Bunsenges. Phys. Chem. 91 (1987) 901

[13] U. Köbler, J. Gmeiner and E. Dormann, J. Magn. Magn. Mat. 69 (1987) 189

[14] G. Sachs, W. Stöcklein, B. Bail, E. Dormann and M. Schwoerer, Chem. Phys. Lett. 89 (1982) 179; W. Höptner, M. Mehring, J.U. v. Schütz, H.C. Wolf, B.S. Morra, V. Enkelmann and G. Wegner, Chem. Phys. 73 (1982) 253

[15] P.A. Lee, T.M. Rice and P.W. Anderson, Phys. Rev. Lett., 31 (1973) 462

[16] D.C. Johnston, Phys. Rev. Lett. 52 (1984) 2049; D.C. Johnston, Solid State Comm. 56 (1985) 439; D.C. Johnston, J.P. Stokes, R.A. Klemm, J. Magn. Magn. Mat. 54 (1986) 1317

[17] G. Sachs, E. Dormann and M. Schwoerer, Solid State Comm. 53 (1985) 73

[18] M.J. Rice and S. Strässler, Solid State Commun. 13 (1973) 125

[19] H.J. Schulz in: D. Jerome, L.G. Caron (Eds.), Low-Dimensional Conductors and Superconductors (Plenum, New York 1987), p. 95

[20] G. Grüner, Phys. Rep. 119 (1985) 117

[21] K. Carneiro in: P. Monceau (Ed.), Electronic Properties of Inorganic Quasi-OneDimensional Compounds II (Reidel, Dordrecht 1985), p. 1

[22] S. Sridhar, D. Reagor and G. Grüner, Phys. Rev. B 34 (1986) 2223

[23] W. Riess, W. Brütting and M. Schwoerer, to be published 


\begin{tabular}{|c||c|c|c|}
\hline $\mathrm{X}^{-}$ & $\mathrm{PF}_{6}^{-}$ & $\mathrm{AsF}_{6}^{-}$ & $\mathrm{SbF}_{6}^{-}$ \\
\hline \hline $\mathrm{a} / \AA$ & $6.61 \pm 0.02$ & $6.58 \pm 0.02$ & $6.62 \pm 0.02$ \\
\hline $\mathrm{b} / \AA$ & $12.57 \pm 0.01$ & $12.63 \pm 0.01$ & $12.73 \pm 0.01$ \\
\hline $\mathrm{c} / \AA$ & $14.77 \pm 0.01$ & $14.89 \pm 0.01$ & $15.09 \pm 0.01$ \\
\hline \hline$T_{P} / \mathrm{K}$ & $182 \pm 1$ & $181 \pm 1$ & $175 \pm 1$ \\
\hline
\end{tabular}

Table 1: Lattice constants of (FA) $)_{2} \mathrm{X}$ for the counterions $\mathrm{X}^{-}=\mathrm{PF}_{6}^{-}, \mathrm{AsF}_{6}^{-}, \mathrm{SbF}_{6}^{-}$at room temperature [7] and the measured phase transition temperatures; a significant shift of the phase transition is observed only with the counterion $\mathrm{X}^{-}=\mathrm{SbF}_{6}^{-}$ 
Figure 1: Crystal structure of $(\mathrm{FA})_{2} \mathrm{PF}_{6}$ at room temperature; top: the aromatic hydrocarbon fluoranthene $\mathrm{C}_{16} \mathrm{H}_{10}$ (H-atoms are omitted); below: projection of the crystal structure on the a-c- and b-c*-plane [7]

Figure 2: Temperature dependence of the dc conductivity $\sigma_{\|}(T)$ of a $(\mathrm{FA})_{2} \mathrm{PF}_{6}$ single crystal along the crystallographic a-axis; the dashed line represents thermally activated conductivity with an activation energy $\Delta(0)=81 \mathrm{meV}$

Figure 3: Temperature dependence of the dc conductivity $\sigma_{\|}(T)$ together with the logarithmic derivative $d\left(\log \sigma_{\|}\right) / d(1 / T)$; the Peierls transition at $T_{P}=182 \mathrm{~K}$ can be clearly identified from the singularity in the derivative

Figure 4: Temperature dependence of the effective fluctuation induced gap $2 \Delta_{e f f}(T)$ for a one-dimensional conductor [16]; the effective gap for $(\mathrm{FA})_{2} \mathrm{PF}_{6}$ can be calculated numerically from the measured $\Delta(0)$ values; $T_{P}^{M F}$ is given by: $2 \Delta(0)=3.52 k_{B} T_{P}^{M F}$

Figure 5: Fit of the Johnston model to the experimental data in the high temperature range of $(\mathrm{FA})_{2} \mathrm{PF}_{6} ; R_{0}=-0.5 \Omega, A=3.0 \times 10^{-3} \Omega \mathrm{K}^{-B}, B=0.8$

Figure 6: Temperature dependence of the mean-field (BCS) energy gap and the real Peierls gap in the ground state of a quasi-one-dimensional conductor [19]

Figure 7: Fit of equation (15) to the experimental data for $(\mathrm{FA})_{2} \mathrm{PF}_{6}$ below the Peierls transition; $A=9.3 \times 10^{-3} \Omega \mathrm{K}^{-B}, B=0.7$

Figure 8: Temperature dependence of the effective and Peierls gap for $(\mathrm{FA})_{2} \mathrm{PF}_{6}$; the Peierls gap vanishes at a temperature $T_{P}^{*}=190 \mathrm{~K}$, which is slightly higher than the observed phase transition temperature $T_{P}=182 \mathrm{~K}$ 
$(\mathrm{FA})_{2} \mathrm{PF}_{6}, \mathrm{~T}=298 \mathrm{~K}$
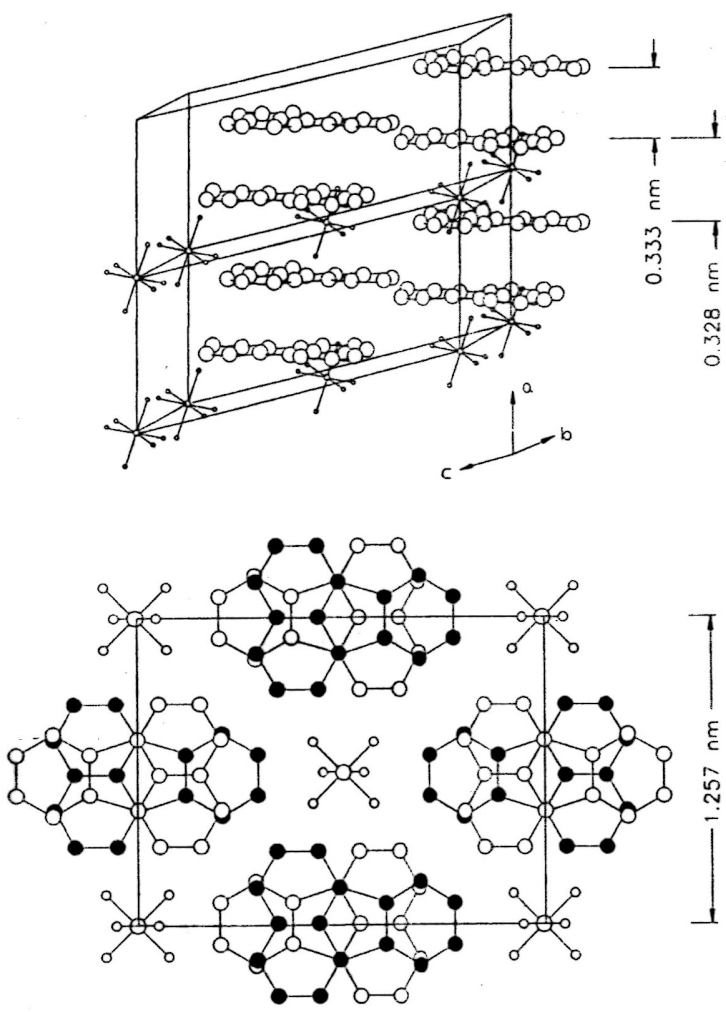


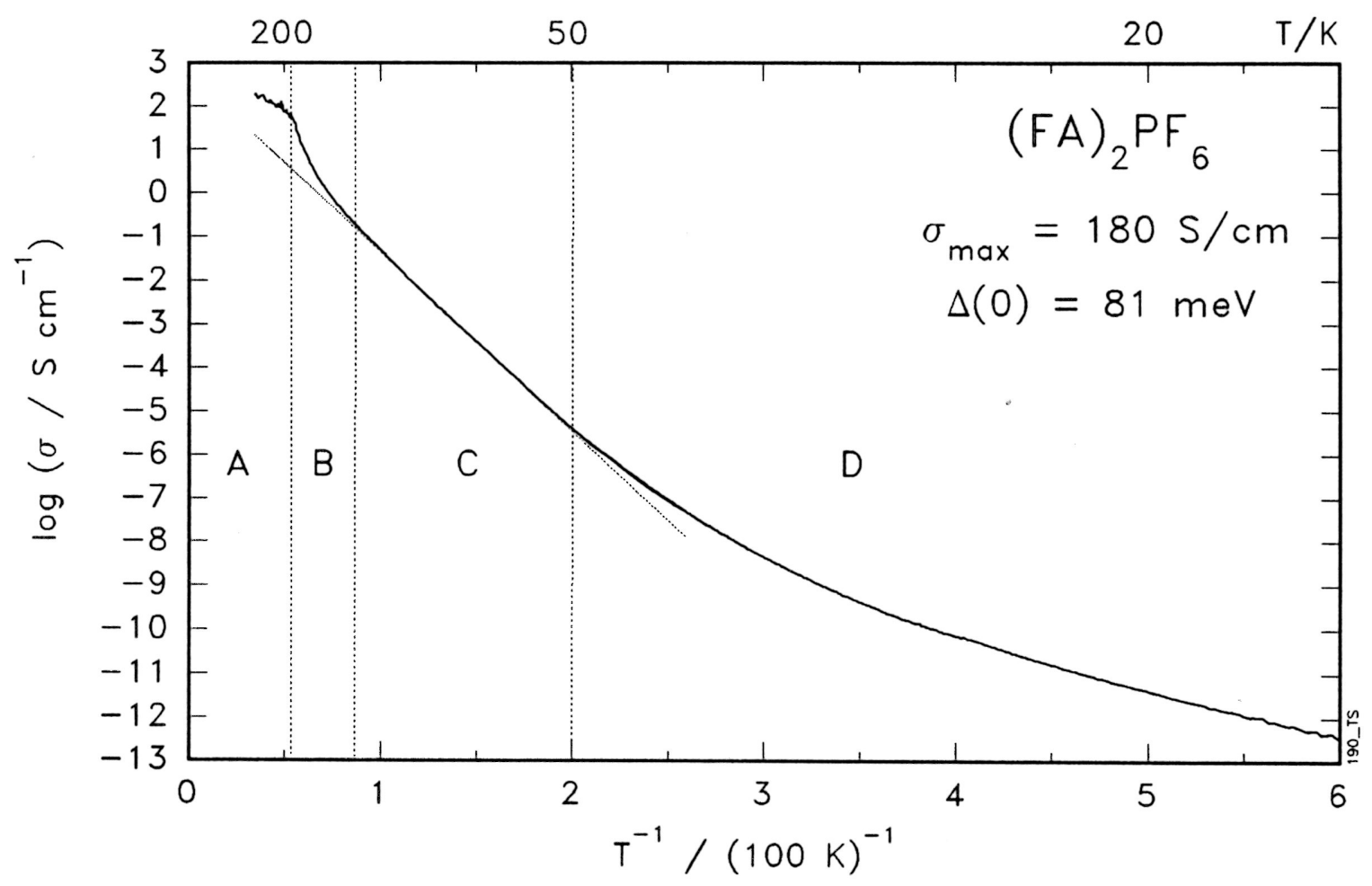




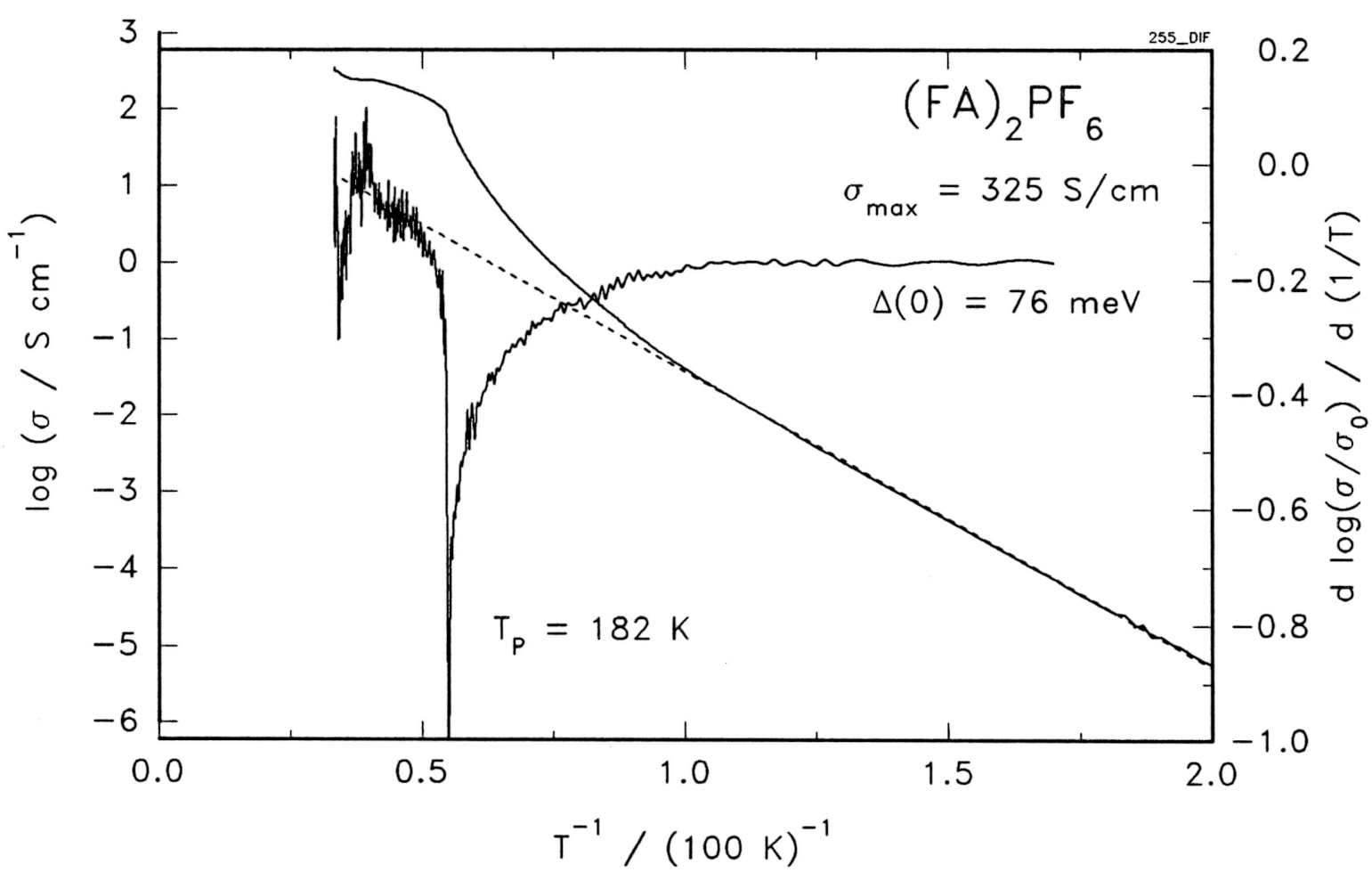




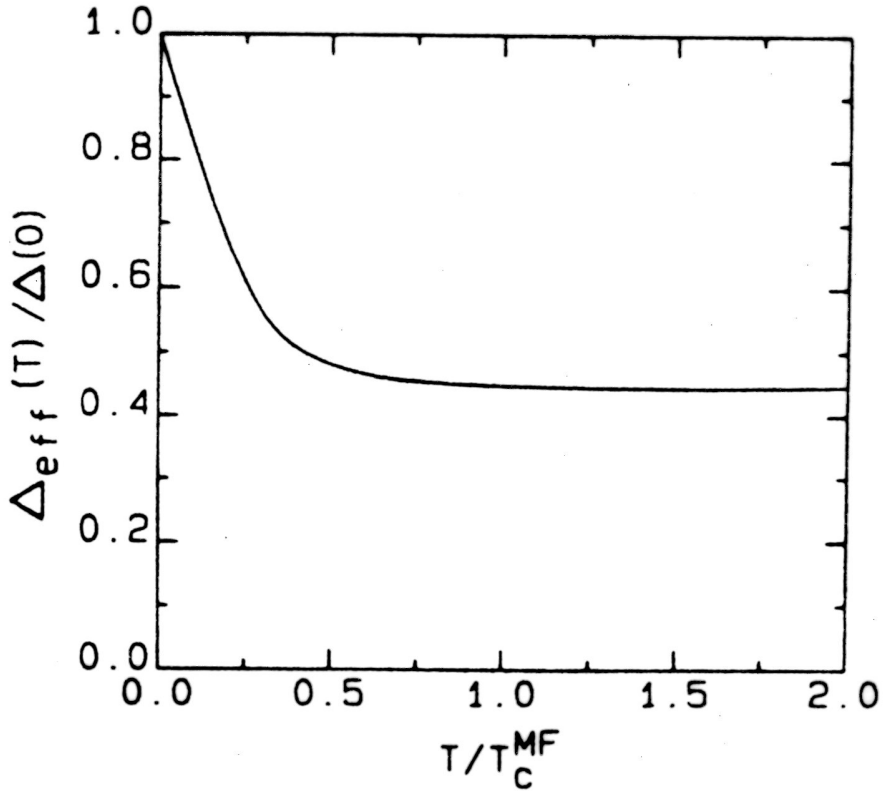




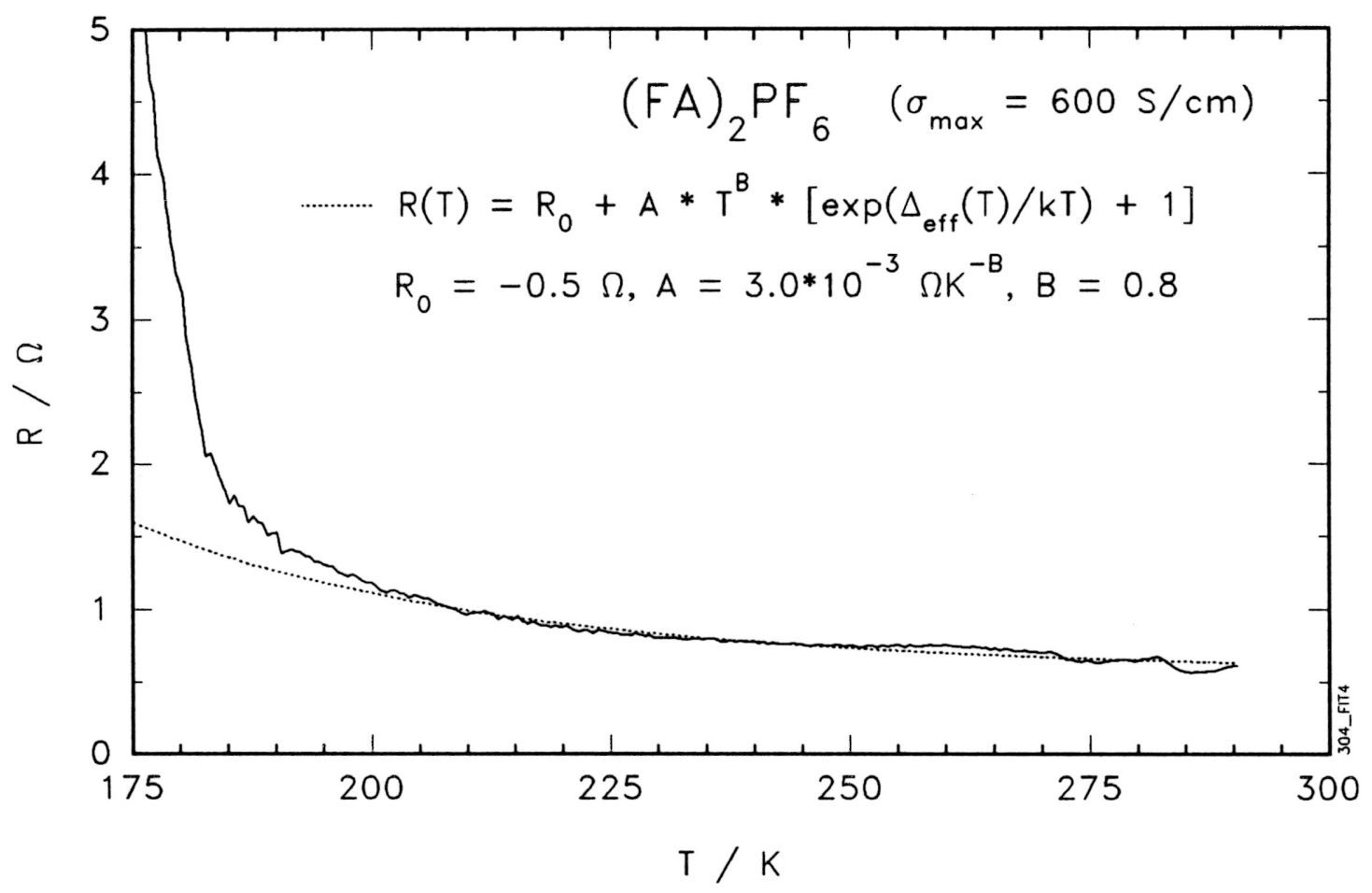




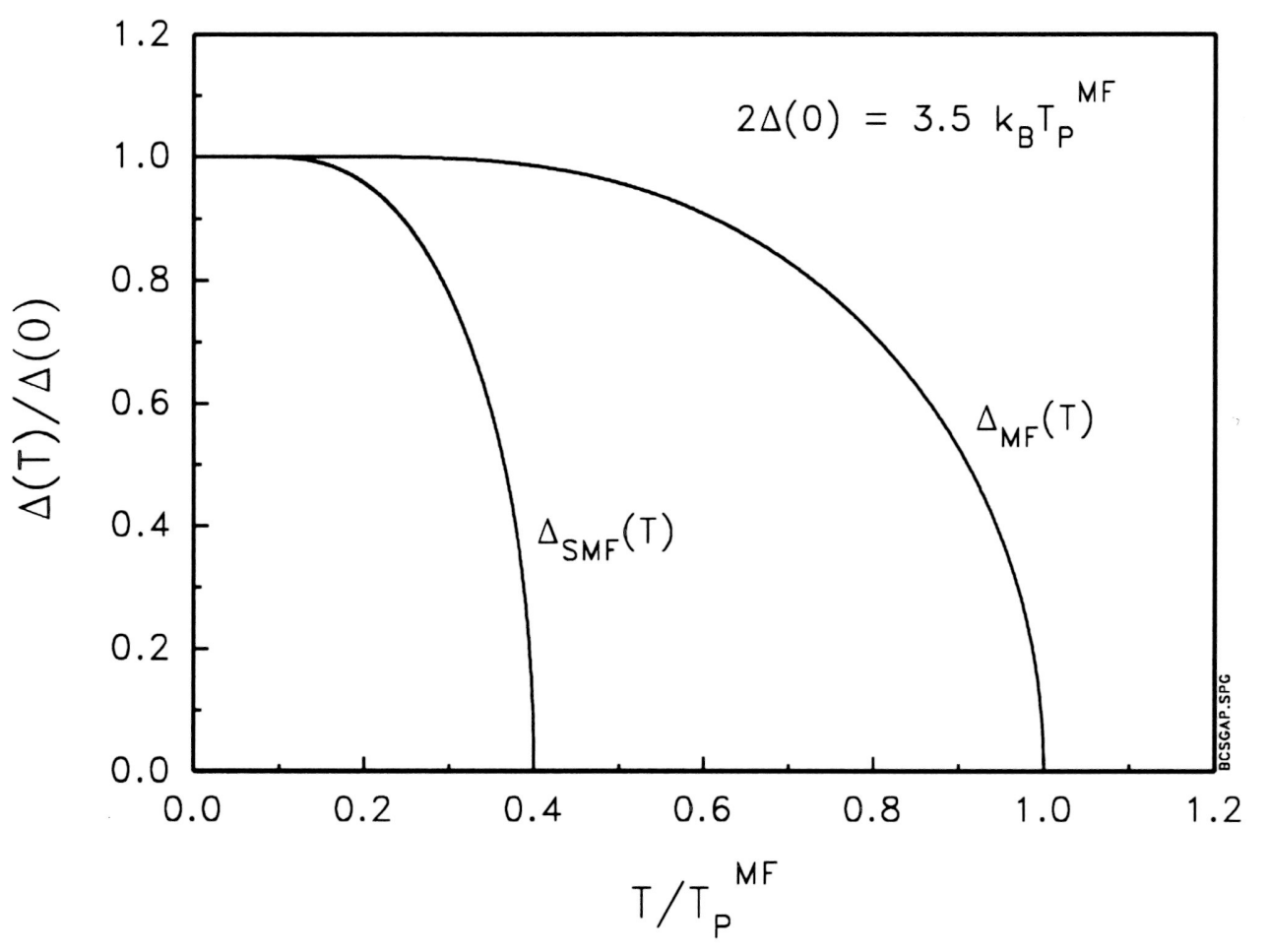




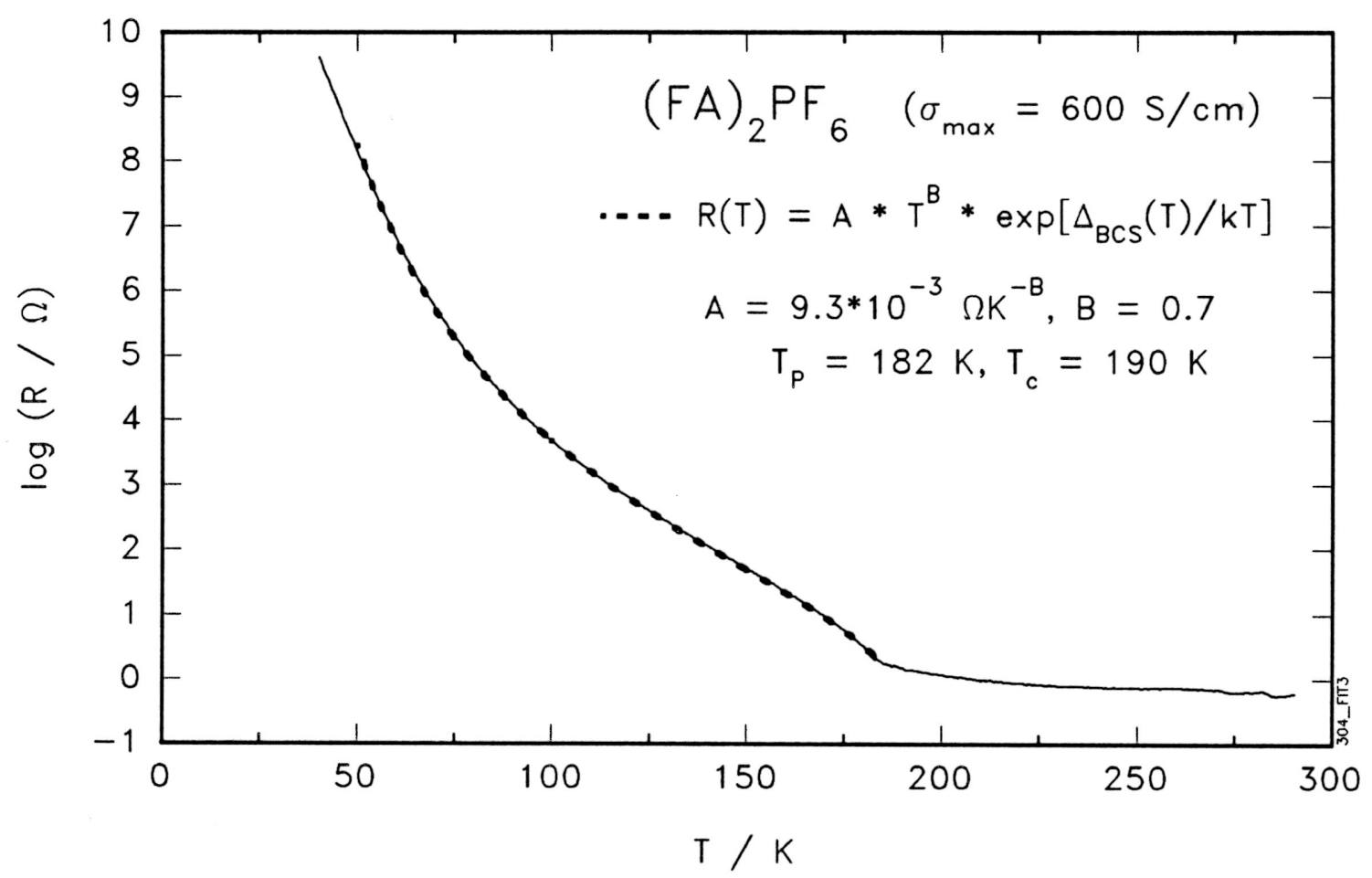




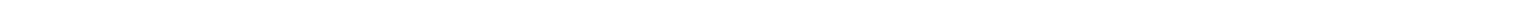

